\title{
A Discussion on the Role of Fermionic Supersymmetric Partners in Lorentz-Symmetry Breaking
}

\author{
José A. Helayël-Neto* \\ Centro Brasileiro de Pesquisas Físicas \\ E-mail: helayelecbpf.br

\section{H. Belich ${ }^{\dagger}$} \\ Universidade Federal do Espírito Santo (UFES), Departamento de Física e Química, Av. \\ Fernando Ferrari S/N, Vitória, ES, CEP 29060-900, Brasil \\ E-mail: belichjregmail.com
}

\section{G. S. Dias}

Instituto Federal do Espírito Santo (IFES), Coordenadoria de Física, Av. Vitória 1729, Jucutuquara, Vitória - ES, 29040-780, CEP 29040-780, Brasil

E-mail: gilmareifes.edu.br

\section{F. J. L. Leal}

Instituto Federal do Espírito Santo (IFES), Coordenadoria de Física, Av. Vitória 1729, Jucutuquara, Vitória - ES, 29040-780, CEP 29040-780, Brasil, and CBPF - Centro Brasileiro de Pesquisas Físicas, Rua Xavier Sigaud 150, Rio de Janeiro, RJ, CEP 22290-180, Brasil E-mail: nandojll@ig.com.br

\section{W. Spalenza}

Instituto Federal do Espírito Santo (IFES) - Campus Cariacica Rod. Gov. José Sette s/n, Cariacica, ES, Brasil, cep 29150-410, Vitória, ES, Brasil

E-mail: spalenzadifes.edu.br

In this work, we present two possible venues to accomodate the $K_{F}$-type Lorentz-symmetry violating Electrodynamics in an $N=1$-supersymmetric framework. A chiral and a vector superfield are chosen to describe the background that signals Lorentz-symmetry breaking. In each case, the $K_{\mu \nu \kappa \lambda}$-tensor is expressed in terms of the components of the background superfield that we choose to describe the breaking. We also present in detail the actions with all fermionic partners of the background that determine $K_{\mu \nu \kappa \lambda}$.

4th International Conference on Fundamental Interactions

August 1-7, 2010

Viçosa, Brazil

\section{* Speaker.}

${ }^{\dagger}$ Also at the Grupo de Física Teórica José Leite Lopes, C.P. 91933, CEP 25685-970, Petrópolis, RJ, and at the International Institute of Physics, Universidade Federal de Rio Grande do Norte, Av. Odilon Gomes de Lima 1722, CEP 59078-400, Natal-RN, Brasil,

${ }^{\ddagger}$ Also at the Department of Physics, University of Alberta, Edmonton, Alberta, Canada T6G 2J1 


\section{Introduction}

The Standard Model Extension (SME) is the natural framework to investigate properties of Lorentz-violation in physical systems involving possible extensions of Higgs mechanism. The SME is by-now a very estimulating research area and a great deal of results have been attained which include bounds on the photon mass [1], radiative corrections [2], systems with fermions [3], neutrinos [4], topological defects [5], topological phases [6], cosmic rays [7], particle decays [8], and other relevant aspects [9], [10]. The SME has also been used as a set-up to propose Lorentz-symmetry violation [11] and CPT- probing experiments [12], which have amounted to the imposition of stringent bounds on the Lorentz-symmetry violating (LV) coefficients.

To take into account how this violation is implemented, in the fermion sector of the SME, for example, there are two CPT-odd terms, $v_{\mu} \bar{\psi} \gamma^{\mu} \psi, b_{\mu} \bar{\psi} \gamma_{5} \gamma^{\mu} \psi$, where $v_{\mu}, b_{\mu}$ are the LV backgrounds [13], [14]. A similar study has also been developed for the case of a non-minimal coupling with the background, with new outcomes [15]. Atomic and optical physics are other areas in which Lorentz-symmetry violation has been intensively studied. Indeed, there are several works examining Lorentz-violation in electromagnetic cavities and optical systems [16], [17], which contributed to establish upper bounds on the LV coefficients.

The appearance of a more complex background, with the existence of an anisotropic vacuum at Planck scale has drawn the attention of particle and field theorists in recent years. Taking strings as the fundamental entities at this level, the idea that Lorentz-symmetry and CPT invariance might be spontaneously broken in some string theories became highlly estimulating [21, 18]. Very recently, the surprising result that CPT-violating neutrino mass-squared difference would be an order of magnitude less than the current uper bound on CPT-violation in the sector of quarks and charged leptons has come to give more support to the proposal of Lorentz-symmetry violation [19], [20]. Actually, the idea of a CPT violation in the neutrino sector had been contemplated by Colladay, Kostelecky and Mewes [21].

Our main motivation to consider a supersymmetric scenario in connection with the violation of Lorentz-symmetry is based on fact that we adopt the viewpoint, according to [18], that the breaking of Lorentz-symmetry may be triggered by the vacuum condensation of a given tensor field. Nevertheless, at this scale where Lorent-symmetry takes place, we may still have supersymmetry (SUSY) or, even if SUSY had already been broken down by some mechanism, supersymmetric partners are present and SUSY imprints are not lost. So, by adopting this scenario, we argue that Lorentz-symmetry violation, as originated at a more fundamental level in the string framework, cannot be dissociated from SUSY. We do not have however elements to decide whether SUSY breaking has occurred before Lorentz-symmetry violation, though the breaking of the latter signals the violation of the former. However, we would like to point out that, in the work of Ref. [32], Katz and Shadmi, present an interesting discussion in the rôle of vacuum expectation values that violate Lorentz symmetry as a possible to realise SUSY breaking by $F$ - and $D$ - terms in the MSSM.

To implement effective Lorentz-symmetry-violating actions in supersymmetric models, one may adopt a superspace formulation with Lorentz and, in some cases, CPT invariances violated by a fixed background in Wess-Zumino-like models [22]. In the gauge sector SUSY-preserving theories where discussed in [23]. Also in the gauge sector, the proposed [25] establishes a supersymmetric minimal extension for the Chern-Simons-like term [24] preserving the usual $(1+3)$-dimensional 
SUSY algebra. The breaking of SUSY will follow the very same route to Lorentz-symmetry breaking: the statement that $v_{\mu}$ is a constant shows thar SUSY is also broken by the fermionic SUSY partner of $v_{\mu}$.

Besides the CPT-odd terms, in the gauge sector, we have the CPT-even part, which is represented by a tensor $K^{\mu v \alpha \beta}$ that presents the same symmetries of the Riemann tensor, as well as an additional double-traceless condition [26]. In this scenario, we present two possibilities of constructing a supersymmetric version for the $K$-type models.

So, we propose to carry out the supersymmetric extension to the bosonic action below:

$$
S=-\frac{1}{4} \int d^{4} x K_{\mu v \kappa \lambda} F^{\mu v} F^{\kappa \lambda}
$$

The "tensor" $K_{\mu v \kappa \lambda}$ it's CPT even, i. e., it does not violate the CPT-symmetry. Despite CPT violation implies violation of Lorentz invariance [30], the reverse is not necesarily true. The action above is Lorentz-violanting in the sense that the "tensor" $K_{\mu \nu \kappa \lambda}$ has a non-zero vacuum expectation value. That "tensor" presents the following symmetries:

$$
K_{\mu v \kappa \lambda}=K_{[\mu v][\kappa \lambda]}, K_{\mu v \kappa \lambda}=K_{\kappa \lambda \mu v}, K_{\mu v}{ }^{\mu v}=0,
$$

as usually appears in the literature, we can reduce the degrees of freedom take into account the ansatz [26]:

$$
\begin{gathered}
K_{\mu v \kappa \lambda}=\frac{1}{2}\left(\eta_{\mu \kappa} \tilde{\kappa}_{v \lambda}-\eta_{\mu \lambda} \tilde{\kappa}_{v \kappa}+\eta_{v \lambda} \tilde{\kappa}_{\mu \kappa}-\eta_{v \kappa} \tilde{\kappa}_{\mu \lambda}\right), \\
\tilde{\kappa}_{\mu v}=\kappa\left(\xi_{\mu} \xi_{v}-\eta_{\mu \nu} \xi^{\alpha} \xi_{\alpha} / 4\right), \\
\kappa=\frac{4}{3} \tilde{\kappa}^{\mu v} \xi_{\mu} \xi_{v},
\end{gathered}
$$

where $\tilde{\kappa}^{\mu v}$ is a traceless "tensor". Using the ansätze (1.3), (1.4), in expression (1.1), we obtain,

$$
S=\frac{\kappa}{4} \int d^{4} x\left\{\frac{1}{2} \xi_{\mu} \xi_{v} F^{\mu}{ }_{\kappa} F^{\kappa v}+\frac{1}{8} \xi_{\lambda} \xi^{\lambda} F_{\mu \nu} F^{\mu v}\right\} .
$$

The supersymmetrization of the action (1.6), instead of (1.1), does not yield higher-spin components in the background dictated by SUSY. To realize the supersymmetrization, we have two possible routs: to achieve supersymmetrisation of (1.1) with $K_{\mu \nu \kappa \lambda}$ given by (1.3), without the particular expression (1.4) for $\tilde{\kappa}_{\mu \nu}$, we get that the background superfield must be chiral. On the other hand, if we are to supersymetrise (1.1) with $K_{\mu \nu \kappa \lambda}$ given by (1.3) and $\tilde{\kappa}_{\mu v}$ given by (1.4), we conclude that a vector superfield must be used to accommodate the background vector, $\xi^{\lambda}$. The content of partners is richer in this case than in the case of the chiral background. The first route accommodate the chiral superfield as $\theta \sigma^{\mu} \bar{\theta} \partial_{\mu} S$, while the second, is carried out by a vector superfield accommodate this "vector" background as $\theta \sigma^{\mu} \bar{\theta} \xi_{\mu}$.

This work is organized as follows: In Section 2, we start with the model of Lorentz breaking proposed as $K_{\mu v \kappa \lambda}$ by Kosteleký. In this Section, we first study the possibility that the background tensor, $K_{\mu \nu \kappa \lambda}$, be originated from a background chiral superfield, which imposes a special form - 
not yet discussed in the literature - for the $K$ - tensor. Then in Section 3, we proceed by investigating the possibility that $K_{\mu \nu \kappa \lambda}$ originates from a (real) vector superfield, which exactly reproduces (1.1) with the $K$ - tensor defined by means of (1.3), and (1.4). Finally, some Concluding Remarks are stated in Section 4.

\section{First Proposal: Lorentz-symmetry Breaking by a Chiral Supermultiplet}

Based on the work of Ref. [25], we take the idea that the background vector could originate from a chiral supermultiplet, $\Omega$. As we shall see, this imposes on $\xi_{\mu}$ the constraint $\xi_{\mu}=\partial_{\mu} S$, where $S$ is a complex scalar. Indeed, as it will become clear at the end of calculations, this choice of SUSY supermultiplet yields an interesting form for $K_{\mu \nu \kappa \lambda}$, completely fixed by a complex scalar field. We then show that the superspace action below shall accomplish the task of yielding the component field extension of the action of eq. (1.6).

Adopting covariant superspace-superfield formulation, we propose the following minimal extension for:

$$
S=\kappa \int d^{4} x d^{2} \theta d^{2} \bar{\theta}\left\{\left(D^{\alpha} \Omega\right) W_{\alpha}\left(\bar{D}_{\dot{\alpha}} \bar{\Omega}\right) \bar{W}^{\dot{\alpha}}+\text { h.c. }\right\} .
$$

The superfields $W_{a}, V, \Omega$ and the SUSY-covariant derivatives $D_{a}, \bar{D}_{\dot{a}}$ hold the definitions:

$$
\begin{aligned}
& D_{a}=\frac{\partial}{\partial \theta^{a}}+i \sigma_{a \dot{a}}^{\mu} \bar{\theta}^{\dot{a}} \partial_{\mu}, \\
& \bar{D}_{\dot{a}}=-\frac{\partial}{\partial \bar{\theta}^{\dot{a}}}-i \theta^{a} \sigma_{a \dot{a}}^{\mu} \partial_{\mu} ;
\end{aligned}
$$

from $\bar{D}_{\dot{b}} W_{a}(x, \theta, \bar{\theta})=0$, and $D^{a} W_{a}(x, \theta, \bar{\theta})=\bar{D}_{\dot{a}} \bar{W}^{\dot{a}}(x, \theta, \bar{\theta})$, it follows that

$$
W_{a}(x, \theta, \bar{\theta})=-\frac{1}{4} \bar{D}^{2} D_{a} V .
$$

Its $\theta$-expansion reads as below:

$$
\begin{aligned}
W_{a}(x, \theta, \bar{\theta}) & =\lambda_{a}(x)+i \theta^{b} \sigma_{b \dot{a}}^{\mu} \bar{\theta}^{\dot{a}} \partial_{\mu} \lambda_{a}(x)-\frac{1}{4} \bar{\theta}^{2} \theta^{2} \square \lambda_{a}(x)+ \\
& +2 \theta_{a} D(x)-i \theta^{2} \bar{\theta}^{\dot{a}} \sigma_{a \dot{a}}^{\mu} \partial_{\mu} D(x)+\sigma^{\mu \nu}{ }_{a}{ }^{b} \theta_{b} F_{\mu v}(x) \\
& -\frac{i}{2} \sigma^{\mu v}{ }_{a}{ }^{b} \sigma_{b \dot{a}}^{\alpha} \theta^{2} \bar{\theta}^{\dot{a}} \partial_{\alpha} F_{\mu v}(x)-i \sigma_{a \dot{a}}^{\mu} \partial_{\mu} \bar{\lambda}^{\dot{a}}(x) \theta^{2}
\end{aligned}
$$

and $V=V^{\dagger}$. The Wess-Zumino gauge choice is taken as usually done:

$$
V_{W Z}=\theta \sigma^{\mu} \bar{\theta} A_{\mu}(x)+\theta^{2} \bar{\theta} \bar{\lambda}(x)+\bar{\theta}^{2} \theta \lambda(x)+\theta^{2} \bar{\theta}^{2} D,
$$

so the action (2.1) is gauge-invariant. The background superfield is so chosen to be a chiral one. Such a constraint restricts the maximum spin component of the background to be an $s=$ $\frac{1}{2}$ component-field, showing up as a SUSY-partner for a spinless dimensionless scalar field. Also, one should notice that $\Omega$ has dimension of mass to -1 . The superfield expansion for $\Omega$ then reads: 


$$
\begin{aligned}
\bar{D}_{\dot{a}} \Omega(x, \theta, \bar{\theta}) & =0, \\
\text { consequently } \Omega(x, \theta, \bar{\theta}) & =S(x)+\sqrt{2} \theta \zeta(x)+i \theta \sigma^{\mu} \bar{\theta} \partial_{\mu} S(x)+ \\
& +\theta^{2} G(x)+\frac{i}{\sqrt{2}} \theta^{2} \bar{\theta} \bar{\sigma}^{\mu} \partial_{\mu} \zeta(x)-\frac{1}{4} \bar{\theta}^{2} \theta^{2} \square S(x) .
\end{aligned}
$$

With its SUSY transformations given by

$$
\begin{aligned}
\delta S & =\sqrt{2} \varepsilon^{\alpha} \zeta_{\alpha}, \\
\delta \zeta_{\alpha} & =\sqrt{2} G \varepsilon_{\alpha}+i \sqrt{2} \sigma_{\alpha \dot{\alpha}}^{\mu} \bar{\varepsilon}^{\dot{\alpha}} \partial_{\mu} S, \\
\delta G & =i \sqrt{2} \bar{\varepsilon}_{\dot{\alpha}} \bar{\sigma}^{\mu \dot{\alpha} \alpha} \partial_{\mu} \zeta_{\alpha} .
\end{aligned}
$$

Notice that, if we wish to have $\partial_{\mu} S$ constant (as we shall get in the sequel, a constant $\partial_{\mu} S$ give us a constant $K_{\mu v \kappa \lambda}$ ), so that $S$ depends lenearly on $x^{\mu}$, SUSY is also broken by the backgroud, as the expression for $\delta \zeta$ shows: if we apply a SUSY transformation on an $\Omega$-superfield for which $S \neq 0, \zeta=0, G=0$, then $\delta \zeta \neq 0$ whenever $\partial_{\mu} S \neq 0$. So, to realise the breaking of Lorentzsymmetry in terms of an $\Omega$ with a constant $\partial_{\mu} S$, then SUSY is not an invariance of the background $\Omega$. However, as it stands, we have an effective model which may descend from a more fundamental theory in which SUSY might be spontaneously broken. At this stage, we have no commitment with any specific mechanism for SUSY breaking.

The component-wise counterpart for the action (2.1) is as follows ${ }^{1}$ :

$$
\begin{aligned}
& S=\kappa \int d^{4} x d^{2} \theta d^{2} \bar{\theta}\left\{\left(D^{\alpha} \Omega\right) W_{\alpha}\left(\bar{D}_{\dot{\alpha}} \bar{\Omega}\right) \bar{W}^{\dot{\alpha}}+\text { h.c. }\right\}=S_{\text {boson }}+S_{\text {fermion }}+S_{\text {coupled }}, \\
& S_{\text {boson }}= \kappa \int d^{4} x\left\{-16\left[\frac{1}{4} \partial_{\lambda} S \partial_{\mu} S^{*}\left(F^{\mu}{ }_{\kappa} F^{\kappa \lambda}+F^{\lambda}{ }_{\kappa} F^{\kappa \mu}\right)+\frac{1}{8} \partial_{\lambda} S \partial^{\lambda} S^{*} F_{\mu \nu} F^{\mu \nu}\right]+\right. \\
&+ 2 D \varepsilon^{\lambda \mu \tau \rho} \partial_{\lambda} S \partial_{\mu} S^{*} F_{\tau \rho}-2 i \partial_{\lambda} S \partial_{\mu} S^{*} \varepsilon^{\lambda \tau \rho \kappa} F_{\tau \rho} F^{\mu}{ }_{\kappa}+8 i D \partial_{\lambda} S \partial_{\mu} S^{*} F^{\lambda \mu}+ \\
&\left.-2 D \partial_{\lambda} S \partial_{\mu} S^{*} \varepsilon^{\lambda \mu \nu \kappa} F_{v \kappa}+8 D^{2} \partial_{\mu} S \partial^{\mu} S^{*}+16 D^{2}|G|^{2}+h . c .\right\} \\
& S_{\text {fermion }}=\kappa \int d^{4} x\left\{\frac{1}{2} \partial_{\lambda} \zeta \sigma^{\mu} \partial_{\mu} \bar{\zeta} \lambda \sigma^{\lambda} \bar{\lambda}+\frac{1}{2} \partial_{\lambda} \zeta \sigma^{\mu} \bar{\lambda} \lambda \sigma^{\lambda} \partial_{\mu} \bar{\zeta}+2 \partial_{\mu} \zeta \partial^{\mu} \lambda \bar{\zeta} \bar{\lambda}+\right. \\
&-\frac{1}{2} \partial_{\lambda} \zeta \sigma^{\lambda} \partial_{\mu} \bar{\zeta} \lambda \sigma^{\mu} \bar{\lambda}-2 \partial_{\lambda} \zeta \sigma^{\lambda} \bar{\sigma}^{\mu} \partial_{\mu} \lambda \bar{\zeta} \bar{\lambda}-\frac{1}{2} \lambda \sigma^{\lambda} \bar{\sigma}^{\mu} \partial_{\lambda} \zeta \bar{\zeta} \partial_{\mu} \bar{\lambda}+ \\
&-\frac{1}{2} \zeta \lambda \bar{\zeta} \square \bar{\lambda}-\zeta \lambda \partial_{\mu} \bar{\zeta} \bar{\sigma}^{\mu} \sigma^{\tau} \partial_{\tau} \bar{\lambda}+\frac{1}{2} \zeta \lambda \partial_{\mu} \bar{\lambda} \bar{\sigma}^{v} \sigma^{\mu} \partial_{\nu} \bar{\zeta}+
\end{aligned}
$$

\footnotetext{
${ }^{1}$ the ref. [31] has been used in our calculations
} 


$$
\begin{aligned}
& +\frac{1}{2} \partial_{\mu} \zeta \sigma^{\mu} \bar{\sigma}^{v} \lambda \bar{\zeta} \partial_{v} \bar{\lambda}-\frac{1}{2 \sqrt{2}} \zeta \square \lambda \bar{\zeta} \bar{\lambda}-\frac{1}{2} \zeta \partial_{v} \lambda \partial_{\mu} \bar{\lambda} \bar{\sigma}^{\mu} \sigma^{v} \bar{\lambda}+ \\
& \left.-\frac{1}{2} \zeta \partial_{v} \lambda \partial_{\mu} \bar{\zeta} \bar{\sigma}^{v} \sigma^{\mu} \bar{\lambda}+\zeta \partial_{\mu} \lambda \bar{\zeta} \partial^{\mu} \bar{\lambda}-2 \zeta \sigma^{\mu} \partial_{\mu} \bar{\lambda} \bar{\zeta} \bar{\sigma}^{v} \partial_{v} \lambda+\text { h.c. }\right\} \\
& S_{\text {coupled }}=\kappa \int d^{4} x\left\{-4 i D \zeta \sigma^{\mu} \partial_{\mu} \bar{\zeta}-2 \sqrt{2} i D G^{*} \zeta \sigma^{\mu} \partial_{\mu} \bar{\lambda}+2 \sqrt{2} D \partial_{v} \lambda \sigma^{v} \bar{\sigma}^{\mu} \zeta \partial_{\mu} S^{*}+\right. \\
& +2 D \zeta \sigma^{v} \partial_{\mu} \bar{\zeta} F_{v}{ }^{\mu}+i D \varepsilon^{\tau \rho \mu \alpha} \zeta \sigma_{\alpha} \partial_{\mu} \bar{\zeta} F_{\tau \rho}+\sqrt{2} G^{*} \zeta \sigma^{\mu} \partial_{v} \bar{\lambda} F_{\mu}{ }^{v}+ \\
& +\frac{i}{\sqrt{2}} G^{*} \varepsilon^{\tau \rho \mu \alpha} \zeta \sigma_{\alpha} \partial_{\mu} \bar{\lambda} F_{\tau \rho}+\sqrt{2} i \zeta \sigma^{\tau} \bar{\sigma}^{v} \partial_{v} \lambda \partial_{\mu} S^{*} F_{\tau}^{\mu}+ \\
& -\frac{1}{\sqrt{2}} \varepsilon^{\tau \rho \mu \alpha} \zeta \sigma_{\alpha} \bar{\sigma}^{v} \partial_{\mu} S^{*} \partial_{v} \lambda F_{\tau \rho}-4 \sqrt{2} i G^{*} D \zeta \sigma^{\mu} \partial_{\mu} \bar{\lambda}+ \\
& +2 \sqrt{2} D \zeta \partial_{\mu} \lambda \partial^{\mu} S^{*}-\frac{i}{\sqrt{2}} \varepsilon^{\mu \nu \kappa \tau} \zeta \partial_{\tau} \lambda \partial_{\mu} S^{*} F_{v \kappa}+\frac{1}{2 \sqrt{2}} \varepsilon^{\mu v \kappa \tau} \zeta \partial_{\tau} \lambda \partial_{\mu} S^{*} F_{v \kappa}+ \\
& -4 i D^{2} \bar{\zeta} \bar{\sigma}^{\mu} \partial_{\mu} \zeta-2 D \bar{\zeta} \bar{\sigma}^{v} \partial_{\mu} \zeta F_{v}{ }^{\mu}+i D \varepsilon^{v \kappa \mu \alpha} \bar{\zeta} \bar{\sigma}_{\alpha} \partial_{\mu} \zeta F_{v \kappa}+ \\
& +2 \sqrt{2} i D G^{*} \partial_{\mu} \zeta \sigma^{\mu} \bar{\lambda}+2 D \partial_{\mu} \zeta \sigma^{\tau} \bar{\zeta} F_{\tau}^{\mu}+i D \varepsilon^{\tau \rho \mu \alpha} \partial_{\mu} \zeta \sigma_{\alpha} \bar{\zeta} F_{\tau \rho}+ \\
& +2 \partial_{\mu} \zeta \sigma^{\tau} \bar{\sigma}^{v \kappa} \bar{\zeta} F_{v \kappa} F_{\tau}{ }^{\mu}+i \varepsilon^{\tau \rho \mu \alpha} \partial_{\mu} \zeta \sigma_{\alpha} \bar{\sigma}^{v \kappa} \bar{\zeta} F_{\tau \rho} F_{v \kappa}+\sqrt{2} \partial_{\mu} \zeta \sigma^{\tau} \bar{\lambda} F_{\tau}{ }^{\mu}+ \\
& +\frac{i}{\sqrt{2}} \varepsilon^{\tau \rho \mu \alpha} \partial_{\mu} \zeta \sigma_{\alpha} \bar{\lambda} F_{\tau \rho}-2 \sqrt{2} i G D \lambda \sigma^{\mu} \partial_{\mu} \bar{\zeta}+2 i|G|^{2} \lambda \sigma^{\mu} \partial_{\mu} \bar{\lambda}+ \\
& -2 i \partial_{v} \lambda \sigma^{v} \bar{\sigma}^{\mu} \lambda \partial_{\mu} S^{*}+4 \sqrt{2} i G D \partial_{\mu} \lambda \sigma^{\mu} \bar{\zeta}-2 \sqrt{2} i G D \bar{\zeta} \bar{\sigma}^{\mu} \partial_{\mu} \lambda \bar{\zeta}+ \\
& -\sqrt{2} G \bar{\zeta} \bar{\sigma}^{\mu} \partial_{\tau} \lambda F_{\mu}{ }^{\tau}+\frac{i}{\sqrt{2}} G \varepsilon^{\mu \nu \tau \alpha} \bar{\zeta} \bar{\sigma}_{\alpha} \partial_{\tau} \lambda F_{\mu \nu}-2 i|G|^{2} \bar{\lambda} \bar{\sigma}^{\mu} \partial_{\mu} \lambda+ \\
& +2 \sqrt{2} D \partial_{\mu} S \bar{\zeta} \partial^{\mu} \bar{\lambda}+\sqrt{2} i \partial_{\mu}(\bar{\lambda} \bar{\zeta}) \partial_{\lambda} S F^{\lambda \mu}-\frac{1}{\sqrt{2}} \varepsilon^{\mu \lambda \tau \rho} \partial_{\mu}(\bar{\lambda} \bar{\zeta}) \partial_{\lambda} S F_{\tau \rho}+ \\
& -2 \sqrt{2} D \bar{\lambda} \partial_{\mu} \bar{\zeta} \partial^{\mu} S+2 \sqrt{2} D \bar{\zeta} \bar{\sigma}^{\mu} \sigma^{v} \partial_{v} \bar{\lambda} \partial_{\mu} S-\sqrt{2} i \bar{\zeta} \bar{\sigma}^{v} \sigma^{\mu} \partial_{\mu} \bar{\lambda} F_{v \lambda} \partial^{\lambda} S+ \\
& -\frac{1}{\sqrt{2}} \varepsilon^{v \kappa \lambda \alpha} \bar{\zeta} \bar{\sigma}_{\alpha} \sigma^{\mu} \partial_{\mu} \bar{\lambda} F_{v \kappa} \partial_{\lambda} S+2 G^{*} \bar{\lambda} \bar{\sigma}^{\mu} \sigma^{v} \partial_{v} \bar{\lambda} \partial_{\mu} S-2 \sqrt{2} \partial_{\mu} S \partial^{\mu} D \bar{\zeta} \bar{\lambda}+ \\
& +\sqrt{2} D \partial_{\nu} \zeta \sigma^{v} \bar{\sigma}^{\mu} \lambda \partial_{\mu} S^{*}-\frac{i}{\sqrt{2}} \bar{\sigma}^{\lambda} \partial_{\lambda} \zeta \lambda \sigma^{v} \partial_{\mu} S^{*} F_{v}{ }^{\mu}+\frac{1}{2 \sqrt{2}} \varepsilon^{\mu \nu \kappa \alpha} \lambda \sigma_{\alpha} \bar{\sigma}^{\lambda} \partial_{\lambda} \zeta \partial_{\mu} S^{*} F_{v \kappa}+ \\
& -\frac{1}{2} \partial_{\lambda} \zeta \sigma^{\lambda} \bar{\lambda} \lambda \sigma^{\mu} \partial_{\mu} \bar{\zeta}+\sqrt{2} D \lambda \sigma^{\lambda} \bar{\sigma}^{\mu} \partial_{\lambda} \zeta \partial_{\mu} S^{*}-\frac{i}{\sqrt{2}} \partial_{\lambda} \zeta \sigma^{v} \bar{\sigma}^{\lambda} \lambda \partial_{\mu} S^{*} F_{v}{ }^{\mu}+ \\
& \left.-\frac{1}{2 \sqrt{2}} \varepsilon^{\mu \nu \kappa \alpha} \partial_{\lambda} \zeta \sigma_{\alpha} \bar{\sigma}^{\lambda} \lambda \partial_{\mu} S^{*} F_{v \kappa}+\text { h.c. }\right\} \text {. }
\end{aligned}
$$

We notice that, in trying to supersymmetrise the $\xi_{\mu} \xi_{v} F_{\kappa}^{\mu} F^{\kappa v}$-term, we automatically get the supersymmetrisation of the term $\xi_{\lambda} \xi^{\lambda} F^{2}$. This is not a simple coincidence, but it can naturally be expected from an analysis of the irreducible representations of $S O(1,3)$. We conclude that, in the case the background superfield is chiral, we are able to write down the supersymmetric version of (1.1) with $K_{\mu v \kappa \lambda}$ given by [26]:

$$
K_{\mu v \kappa \lambda}=-16\left(\eta_{k \mu} \tilde{\kappa}_{v \lambda}-\eta_{\mu \lambda} \tilde{\kappa}_{v \kappa}-\eta_{v \lambda} \tilde{\kappa}_{\mu \kappa}-\eta_{k v} \tilde{\kappa}_{\mu \lambda}\right)
$$


with,

$$
\tilde{\kappa}_{\mu v}=\kappa\left\{\left(\frac{\partial_{\mu} S \partial_{v} S^{*}+\partial_{v} S \partial_{\mu} S^{*}}{2}\right)-\partial_{\lambda} S \partial^{\lambda} S^{*} \eta_{\mu v} / 4\right\}
$$

and

$$
K_{\mu v}^{\mu v}=0 .
$$

Concluding, this special form for $\tilde{\kappa}$ is a natural consequence of the assumption that a chiral superfield carries the background that breaks Lorentz-symmetry.

It is worthy to notice that $\tilde{\kappa}$, and consequently $K$, depend exclusively on the scalar component $S$. No $\zeta$-condensate and no $G$-dependence appear in the expression for the $K$-tensor. Since the action (2.9) is quadratic in $\Omega$, one might in principle expect that tensor bilinears in $\zeta$ could show up as contributions to $K_{\mu v \kappa \lambda}$. However, the $K$-tensor depends exclusively on the gradient of $S$. It becomes clear that a constant $\partial_{\mu} S$ ensures the constancy of $K_{\mu \nu \kappa \lambda}$, as we had already anticipated. In a particular case, where the background fields $\partial_{\mu} S \neq 0, \zeta=0$, and $G=0$, we have that the auxiliar field, $D$, is given by (we suppose the supersymmetric version of the $F_{\mu \nu}^{2}$-term is added up),

$$
D=-\frac{8 \kappa i \partial_{\mu} S \partial_{\nu} S^{*} F^{\mu \nu}}{16+32 \kappa \partial_{\mu} S \partial^{\mu} S^{*}}
$$

It is interesting to comment that, by virtue of (2.16), which is valid in the conditions above for the background, the breaking of Lorentz-symmetry, fixes the auxiliary field to be non-trivial, even, if the gauge potential superfield $(V)$ is not coupled to matter. The backgound, as (2.16) shows, determines $D$ by the gauge field strenght, $F_{\mu \nu}$. However, if supersymmetric matter happens to be minimally coupled to the gauge field, then (2.16) indicates that charged scalar particles (selectrons, for example) may acquire a magnetic dipole moment given in terms of the vector $\vec{\mu}=\vec{v} \times \vec{v}^{*}$, where $\vec{v} \equiv \vec{\nabla} S$. This investigation is being pursued and we shall soon report on it [29].

\section{Second Proposal: Lorentz-symmetry Breaking from a Vector Supermultiplet}

Adopting covariant superspace-superfield formulation, we propose the following minimal extension of (1.6) for:

$$
S=\kappa \int d^{4} x d^{2} \theta d^{2} \bar{\theta}\left\{\left(D^{\alpha} \Xi\right) W_{\alpha}\left(\bar{D}_{\dot{\alpha}} \Xi\right) \bar{W}^{\dot{\alpha}}+\text { h.c. }\right\} .
$$

$W_{\alpha}$ is the superfield strenght of the gauge supermultiplet $V_{W Z}$, as given in eq. (1.1). $\Xi$ is the so-called vector superfield, whose $\theta$ - expansion is as follows:

$$
\Xi(\theta, \bar{\theta})=C+\theta \chi+\bar{\theta} \bar{\chi}+\theta^{2} M+\bar{\theta}^{2} M^{*}+\theta \sigma^{\mu} \bar{\theta} \xi_{\mu}+\theta^{2} \bar{\theta} \bar{\psi}+\bar{\theta}^{2} \theta \psi+\theta^{2} \bar{\theta}^{2} B
$$

In the special case of constant background component fields, the SUSY transformations simplify and acquire the form below: 


$$
\begin{aligned}
\delta C & =\varepsilon^{\alpha} \chi_{\alpha}+\bar{\varepsilon}_{\dot{\alpha}} \bar{\chi}^{\dot{\alpha}} \\
\delta \chi_{\alpha} & =2 M \varepsilon_{\alpha}+\sigma_{\alpha \dot{\alpha}}^{\mu} \bar{\varepsilon}^{\dot{\alpha}} \xi_{\mu} \\
\delta \bar{\chi}^{\dot{\alpha}} & =-\varepsilon^{\alpha} \sigma_{\alpha \dot{\beta}}^{\mu} \varepsilon^{\dot{\beta} \dot{a}} \xi_{\mu}+2 M^{*} \bar{\varepsilon}^{\dot{\alpha}} \\
\delta M & =\bar{\varepsilon}_{\dot{\alpha}} \bar{\psi}^{\dot{\alpha}} \\
\delta M^{*} & =\varepsilon^{\alpha} \chi_{\alpha} \\
\delta \xi^{\mu} & =\varepsilon^{\alpha} \sigma_{\alpha \dot{\alpha}}^{\mu} \bar{\psi}^{\dot{\alpha}}-\bar{\varepsilon}_{\dot{\alpha}} \bar{\sigma}^{\mu \dot{\alpha} \alpha} \psi_{\alpha}, \\
\delta \bar{\psi}^{\dot{\alpha}} & =2 \bar{\varepsilon}^{\dot{\alpha}} B \\
\delta \psi_{\beta} & =2 \varepsilon_{\beta} B \\
\delta B & =0 .
\end{aligned}
$$

In the general case, $B$ transforms as a total derivative; only for a constant $\psi$-background, we get $\delta B=0$. Notice that we are taking the full $\theta$-expansion for $\Xi$. Nothing like a Wess-Zumino gauge can be taken, for $\Xi$ is a fixed background and is not a gauge super-potential.

The superaction in component fields is given by,

$$
\begin{aligned}
& S=\kappa \int d^{4} x d^{2} \theta d^{2} \bar{\theta}\left\{\left(D^{\alpha} \Xi\right) W_{\alpha}\left(\bar{D}_{\dot{\alpha}} \Xi\right) \bar{W}^{\dot{\alpha}}+\text { h.c. }\right\}=S_{\text {boson }}+S_{\text {fermion }}+S_{\text {coupled }} \\
& S_{\text {boson }}=\kappa \int d^{4} x\left\{2\left(\frac{1}{2} \xi_{\lambda} \xi_{\mu} F^{\mu}{ }_{\kappa} F^{\kappa \lambda}+\frac{1}{8} \xi_{\lambda} \xi^{\lambda} F_{\mu \nu} F^{\mu \nu}\right)+\right. \\
&\left.+\frac{i}{4} \xi_{\lambda} \xi_{\mu} \varepsilon^{\lambda \tau \rho \kappa} F_{\tau \rho} F^{\mu}{ }_{\kappa}-2 D^{2} \xi_{\lambda} \xi^{\lambda}+8|M|^{2} D^{2}+\text { h.c. }\right\}
\end{aligned}
$$

where we can readily identify the action (1.1) with $K_{\mu \nu \kappa \lambda}$ given as in (1.3), and (1.4).

$$
\begin{aligned}
S_{\text {fermion }} & =\kappa \int d^{4} x\left\{-i \psi \sigma^{\mu} \partial_{\mu} \bar{\lambda} \bar{\chi} \bar{\lambda}+\psi \lambda \bar{\psi} \bar{\lambda}+\frac{i}{2} \partial_{\mu} \lambda \sigma^{\mu} \bar{\psi} \bar{\chi} \bar{\lambda}+\psi \lambda \partial_{\mu} \lambda \sigma^{\mu} \bar{\chi}+\right. \\
& \left.-\frac{i}{2} \lambda \sigma^{\mu} \bar{\psi} \bar{\chi} \partial_{\mu} \bar{\lambda}+\lambda^{2} \bar{\psi} \bar{\lambda}+i \chi \lambda \partial_{\mu} \lambda \sigma^{\mu} \bar{\psi}-i \chi \lambda \psi \sigma^{\mu} \partial_{\mu} \bar{\lambda}+h . c .\right\} \\
S_{\text {coupled }} & =\kappa \int d^{4} x\left\{-\frac{3}{4} i \chi \lambda \xi_{\mu} \partial^{\mu} D-\frac{1}{2} \chi \lambda \xi_{\mu} \partial_{v} F^{v}{ }_{\mu}+\right. \\
& -\frac{i}{2} \chi \lambda \varepsilon^{\psi v \kappa \mu} \xi_{\mu} \partial_{\psi} F_{v \kappa}+4 \chi \lambda B D+D \chi \sigma^{\mu} \bar{\chi} \partial_{v} F^{v}{ }_{\mu}+ \\
& +\frac{i}{4} D \varepsilon^{\psi v \kappa \alpha} \chi \sigma_{\alpha} \bar{\chi} \partial_{\psi} F_{v \kappa}-6 i D M^{*} \chi \sigma^{\mu} \partial_{\mu} \bar{\lambda}+i D \partial_{v} \lambda \sigma^{v} \bar{\sigma}^{\mu} \chi \xi_{\mu}+ \\
& -4 D^{2} \chi \psi-\frac{i}{2} \chi \sigma^{\tau} \bar{\chi} F_{\tau \rho} \partial_{v} F^{v \rho}+\frac{1}{4} \varepsilon^{\tau \rho \kappa \alpha} \chi \sigma_{\alpha} \bar{\chi} F_{\tau \rho} \partial_{v} F^{v}{ }_{\kappa}+
\end{aligned}
$$




$$
\begin{aligned}
& +\frac{1}{4} \varepsilon^{\lambda v \kappa \rho} \chi \sigma^{\tau} \bar{\chi} F_{\tau \rho} \partial_{\lambda} F_{v \kappa}+\frac{i}{8} \varepsilon^{\psi v \kappa \alpha} \varepsilon_{\alpha \beta}^{\tau \rho} \chi \sigma^{\beta} \bar{\chi} F_{\tau \rho} \partial_{\psi} F_{v \kappa}+ \\
& +M^{*} \chi \sigma^{\tau} \partial_{v} \bar{\lambda} F_{\tau}{ }^{v}+\frac{i}{2} M^{*} \varepsilon^{\tau \rho \mu \alpha} \chi \sigma_{\alpha} \partial_{\mu} \bar{\lambda} F_{\tau \rho}+\frac{1}{2} \chi \sigma^{\tau} \bar{\sigma}^{v} \partial_{v} \lambda \xi_{\mu} F_{\tau}^{\mu}+ \\
& +\frac{i}{4} \varepsilon^{\tau \rho \mu \alpha} \sigma_{\alpha} \bar{\sigma}^{v} \partial_{v} \lambda \xi_{\mu} F_{\tau \rho}-2 D \chi \sigma^{\tau \rho} \lambda F_{\rho \tau}+\frac{1}{2} \chi \partial_{\mu} \lambda \bar{\chi} \partial^{\mu} \bar{\lambda}+ \\
& +i \chi \partial_{\mu} \lambda \xi^{\mu}-\bar{\chi} \bar{\sigma}^{v} \chi \partial_{\mu} D F_{v}{ }^{\mu}+\frac{i}{2} \varepsilon^{v \kappa \mu \alpha} \bar{\chi} \bar{\sigma}_{\alpha} \chi \partial_{\mu} D F_{v \kappa}+ \\
& +i M D \partial_{\mu} \lambda \sigma^{\mu} \bar{\chi}+\frac{i}{2} M \lambda \sigma^{\kappa} \bar{\chi} \partial_{\mu} F^{\mu}{ }_{\kappa}-\frac{1}{4} M \varepsilon^{\mu v \kappa \alpha} \lambda \sigma_{\alpha} \bar{\chi} \partial_{\mu} F_{v \kappa}+ \\
& -i|M|^{2} \lambda \sigma^{\mu} \partial_{\mu} \bar{\lambda}+i M \partial_{v} \lambda \sigma^{v} \bar{\sigma}^{\mu} \lambda \xi_{\mu}-4 M D \lambda^{2}+ \\
& -\frac{1}{2} \bar{\chi} \bar{\sigma}^{v} \partial_{\mu} \lambda F_{v}{ }^{\mu}+\frac{i}{4} \varepsilon^{v \kappa \mu \alpha} \bar{\chi} \bar{\sigma}_{\alpha} \partial_{\mu} \lambda F_{v \kappa}+\frac{i}{2} M^{*} \partial_{\mu} \lambda \sigma^{\mu} \bar{\lambda}+ \\
& +i \lambda \sigma^{\mu} \partial_{v} \bar{\lambda} \xi^{v} \xi_{\mu}-\frac{i}{2} \lambda \sigma^{\mu} \partial_{\mu} \bar{\lambda} \xi_{\lambda} \xi^{\lambda}-\frac{1}{2} \varepsilon^{\lambda v \mu \alpha} \lambda \sigma_{\alpha} \partial_{v} \bar{\lambda} \xi_{\lambda} \xi_{\mu}+ \\
& +\frac{1}{2} \varepsilon^{v \kappa \lambda \alpha} \lambda \sigma_{\alpha} \bar{\psi} \xi_{\lambda} F_{v \kappa}-B \lambda \sigma^{\mu} \bar{\lambda} \xi_{\mu}+\bar{\chi} \partial_{v} \bar{\lambda} \xi_{\mu} F^{\mu v}+ \\
& +\frac{i}{2} \varepsilon^{\tau \rho v \lambda} \bar{\chi} \partial_{v} \bar{\lambda} \xi_{\lambda} F_{\tau \rho}-\frac{i}{2} \bar{\lambda} \bar{\sigma}^{\rho} \lambda \xi_{\lambda} F_{\rho}^{\lambda}-\frac{1}{4} \varepsilon^{\lambda \tau \rho \alpha} \bar{\lambda} \bar{\sigma}_{\alpha} \lambda \xi_{\lambda} F_{\tau \rho}+ \\
& -i D \partial_{\mu} \bar{\lambda} \bar{\sigma}^{\mu} \sigma^{\lambda} \bar{\chi} \xi_{\lambda}+\frac{1}{2} \partial_{\mu} \bar{\lambda} \bar{\sigma}^{\mu} \sigma^{\kappa} \bar{\chi} \xi_{\lambda} F^{\lambda}{ }_{\kappa}+\frac{i}{4} \varepsilon^{\lambda v \kappa \alpha} \partial_{\mu} \bar{\lambda} \bar{\sigma}^{\mu} \sigma_{\alpha} \bar{\chi} \xi_{\lambda} F_{v \kappa}+ \\
& -i M^{*} \partial_{\mu} \bar{\lambda} \bar{\sigma}^{\mu} \sigma^{\lambda} \bar{\lambda} \xi_{\lambda}-\frac{1}{2} \bar{\chi} \bar{\lambda} \xi_{\lambda} \partial_{v} F^{\lambda v}-\frac{i}{4} \bar{\chi} \bar{\lambda} \varepsilon^{\tau \rho v \lambda} \xi_{\lambda} \partial_{v} F_{\tau \rho}+ \\
& -\frac{1}{2} D^{2} \bar{\chi} \bar{\psi}+D \bar{\chi} \bar{\sigma}^{v \kappa} \bar{\psi} F_{v \kappa}-2 M^{*} D \bar{\psi} \bar{\lambda}-\frac{i}{2} \psi \sigma^{\tau} \bar{\lambda} \xi_{\mu} F_{\tau}{ }^{\mu}+ \\
& +\frac{1}{4} \varepsilon^{\tau \rho \mu \alpha} \psi \sigma_{\alpha} \bar{\lambda} \xi_{\mu} F_{\tau \rho}-B \lambda \sigma^{\mu} \bar{\lambda} \xi_{\mu}+4 B D \bar{\chi} \bar{\lambda}+h . c . \\
&
\end{aligned}
$$

The terms in the superactions (2.9) and (3.4) have the same form with the factor $\left(D^{\alpha} \Omega\right) W_{\alpha}$ replaced by $\left(D^{\alpha} \Xi\right) W_{\alpha}$. Then, we obtain the right combination, i. e., $\frac{1}{2} \xi_{\mu} \xi_{v} F^{\mu}{ }_{\kappa} F^{\kappa v}+\frac{1}{8} \xi_{\lambda} \xi^{\lambda} F_{\mu v} F^{\mu v}$. To get the term, $\xi_{\mu} \xi_{v} F^{\mu}{ }_{K} F^{\kappa v}$, we could also propose the action below:

$$
S=\kappa \int d^{4} x d^{2} \theta d^{2} \bar{\theta}\left\{\left(D^{\alpha} \Xi\right)\left(D_{\alpha} \Xi\right) W^{\beta} W_{\beta}+\text { h.c. }\right\}
$$

Also in this case, when we supersymmetrise the $\xi_{\mu} \xi_{v} F^{\mu}{ }_{\kappa} F^{\kappa v}$-term, automatically comes out $\xi_{\lambda} \xi^{\lambda} F^{2}$. So, we have a second way to build up the supersymmetric extension of the term $\left(\frac{1}{2} \xi_{\mu} \xi_{v} F^{\mu}{ }_{\kappa} F^{\kappa v}+\frac{1}{8} \xi_{\lambda} \xi^{\lambda} F_{\mu \nu} F^{\mu v}\right)$. We just mention this possible second way of working out the supersymmetrisation of (1.6) by a vector superfield background, but we do not exploit here this second possibility. This is why we do not project the superfield action (3.8) into components.

Though we formulate our model in terms of superspace and superfields, the tensor calculus of supersymmetry, we would like to point out that with a Lorentz-symmetry breaking, in the chiral case given by $\partial_{\mu} S \neq 0$, in the vector case parametrized by $\xi^{\lambda} \neq 0$, SUSY is readily seen to be broken by the background. But, as it was emphasized, we do not have elements to decide whether SUSY breaking has occurred before Lorentz-symmetry violation, though the breaking of the latter signals the violation of the former. 


\section{Concluding Comments}

Our effort in this paper has mainly consisted in finding out possible $N=1$ supersymmetric scenarios for the $K$ - tensor- realized Lorentz-symmetry breaking considered in the literature ( see the Refs. quoted throughout the present work).

We propose two viable descriptions. The first approach is based on a chiral superfield that accommodates the content of the background responsible for the breaking of Lorentz-symmetry. In this case, a complex scalar is the source for $K_{\mu \nu \kappa \lambda}$. The constancy of the $K-$ tensor is ensured by the linear dependence of $S$ on the $x^{\mu}$ - coordinates. An interesting question to be investigated is the analysis of the photon - photino mass splitting in terms of the background field components, specially if the fermion component $\zeta$ is a non-vanishing constant background. From the expression for $S_{\text {coupled }}$ in eq. (2.12), there result interesting terms that mix the gauge superfield components $\left(A^{\mu}, \lambda\right.$, and $\left.D\right)$ in bilinear terms where the (constant) background fields are also present, as mass parameters. The task of getting the $\left\langle A_{\mu} A_{\nu}>-<A_{\mu} \lambda>-<\lambda \lambda>-<\lambda D>-\right.$ and $\langle D D>$ - propagators demands special technicalities ( Fierzings and spin-projection operators) and, once these tree level 2- point functions are worked out, we can read off the propagator poles and discuss in terms of the background components $(S, \zeta$, and $G$ ). The rôle of the background fermionic condensates become clear after the propagator poles are identified.

This discussion holds through also in the cases of a vector-superfield background that carries $\xi_{\mu}$, from which $K_{\mu \nu \kappa \lambda}$ is expressed. This case exhibits a richer background and fermion condensates that mix $\chi$ and $\Psi$ ( see eq. (3.7)), in addition to the $\chi$ - and $\Psi$-condensates become important.

So, to our mind, in either case, the important question that our study may raise concerns the influence of the Lorentz-symmetry violating background on the spectrum and which restrictions it should have so as to avoid the appearance of non-physical excitations, such as tachyons and ghosts.

Finally, a non-trivial question that remains to be addressed to is the relation between SUSY and Lorentz-symmetry breakings. We treat the latter in a supersymmetric formulation and, as we have previously commented, our backgrounds (both $\Omega$ and $\Xi$ ) are not invariant under SUSY. They simply express the fact that Lorentz-symmetry breaking does not support exact SUSY. However, by no means, we are stating that we break Lorentz-symmetry and SUSY at the same time. SUSY could have been broken before, by some more fundamental mechanism, and Lorentz-symmetry breaking takes place in an environment which still keeps the inheritance of SUSY through the whole set of supersymmetric partners. This is why approach the deviation from Lorentz-symmetry in connection with SUSY. However, it would be an interesting task- and we shall soon concentrate on this point - to build up a model such that, whenever SUSY is spontaneously broken, some tensor field (that belongs to some supermultiplet of the model coupled to the superfield responsible for the breaking of SUSY) also acquires a non-trivial vacuum expectation value and the Lorentz group simultaneously undergoes spontaneous symmetry breakdown. This sets out another possibility, with a contemporary breaking of SUSY and Lorentz-symmetry. We are considering this situation and we shall report on it elsewhere. 


\section{Acknowledgments}

The authors F. J. L. Leal and J. A. Helayël-Neto thank the Brazilian funding agencies, CNPq and FAPERJ, for financial support.

\section{References}

[1] H. Belich M. M. Ferreira Jr, J.A. Helayel-Neto, M. T. D. Orlando, Phys. Rev. D 67, 125011 (2003); -ibid, Phys. Rev. D 69, 109903 (E) (2004); H. Belich, M. M. Ferreira Jr, J.A. Helayel-Neto, M. T. D. Orlando, Phys. Rev. D 68, 025005 (2003); A. P. Baêta Scarpelli, H. Belich, J. L. Boldo, J.A. Helaeyl-Neto, Phys. Rev. D 67, 085021 (2003); H. Belich et al., Nucl. Phys. B Suppl. 127, 105 (2004); H. Belich, M. M. Ferreira Jr and J. A. Helayel-Neto, Eur. Phys. J. C 38, 511 (2005); H. Belich, T. Costa-Soares, M.M. Ferreira Jr., J. A. Helayël-Neto, Eur. Phys. J. C 42, 127 (2005), Cantcheff M. B Eur. Phys. J. C 46, 247 - 254, (2006), H. Belich, et al. REV. BRAS. ENS. FIS. 29, 57 (2007), H. Belich, L. P. Colatto, T. Costa-Soares, J. A. Helayël-Neto, M. T. D. Orlando Eur. Phys. J. C 62, 425-432 (2009).

[2] R. Jackiw and V. A. Kostelecký, Phys. Rev. Lett. 82, 3572 (1999); J. M. Chung and B. K. Chung Phys. Rev. D 63, 105015 (2001); J.M. Chung, Phys.Rev. D 60, 127901 (1999); G. Bonneau, Nucl.Phys. B 593, 398 (2001); M. Perez-Victoria, Phys. Rev. Lett. 83, 2518 (1999); M. Perez-Victoria, JHEP 0104, 032 (2001); O.A. Battistel and G. Dallabona, Nucl. Phys. B 610, 316 (2001); O.A. Battistel and G. Dallabona, J. Phys. G 28, L23 (2002); J. Phys. G 27, L53 (2002); A. P. Baêta Scarpelli, M. Sampaio, M. C. Nemes, and B. Hiller, Phys. Rev. D 64, 046013 (2001); F.A. Brito, T. Mariz, J.R. Nascimento, E. Passos, R.F. Ribeiro, JHEP 0510 (2005) 019.

[3] B. Altschul, Phys. Rev. D 70, 056005 (2004); G. M. Shore, Nucl. Phys. B 717, 86 (2005); D. Colladay and V. A. Kostelecky, Phys. Lett. B 511, 209 (2001); M. M. Ferreira Jr, Phys. Rev. D 70, 045013 (2004); M. M. Ferreira Jr, Phys. Rev. D 71, 045003 (2005); M. M. Ferreira Jr and M. S. Tavares, Int. J. Mod. Phys. A 22, 1685 (2007);J. Alfaro, A.A. Andrianov, M. Cambiaso, P. Giacconi, R. Soldati, Phys. Lett. B 639 (2006) 586-590.

[4] V. Barger, S. Pakvasa, T.J.Weiler, and K.Whisnant, Phys. Rev. Lett. 85, 5055 (2000); V. A. Kostelecky and M. Mewes, Phys. Rev. D 69, 016005 (2004); Phys. Rev. D 70, 031902 (R) (2004); Phys. Rev. D 70, 076002 (2004); T. Katori, A. Kostelecky, R. Tayloe, Phys.Rev. D 74, 105009 (2006); R. Brustein, D. Eichler, S. Foffa, Phys.Rev. D 65, 105006 (2002); Y. Grossman,C. Kilic, J. Thaler, and D.G. E. Walker, Phys. Rev. D 72, 125001 (2005); L. B. Auerbach et al., Phys. Rev. D 72, 076004 (2005); D. Hooper, D. Morgan, and E. Winstanley, Phys. Rev. D 72, 065009 (2005).

[5] M. Lubo, Phys. Rev. D 71, 047701 (2005); M.N. Barreto, D. Bazeia, and R. Meneses, Phys. Rev. D 73, 065015 (2006).

[6] H. Belich, T. Costa-Soares, M.M. Ferreira Jr., J. A. Helayël-Neto, M.T. D. Orlando, Phys. Lett. B 639, 678 (2006); H. Belich, T. Costa-Soares, M.M. Ferreira Jr., J. A. Helayël-Neto, Eur. Phys. J. C 41, 421 (2005).

[7] O. Gagnon and G. D. Moore, Phys. Rev. D 70, 065002 (2004); J.W. Moffat, Int. J. Mod. Phys. D 12 1279 (2003); F. W. Stecker and S.T. Scully, Astropart. Phys. 23, 203 (2005); F. W. Stecker and S.L. Glashow, Astropart. Phys. 16, 97 (2001).

[8] E. O. Iltan, Eur. Phys. J. C 40, 269 (2005); Mod. Phys. Lett. A19, 327 (2004); JHEP 0306 (2003) 016.

[9] V.A. Kostelecky and R. Lehnert, Phys. Rev. D 63, 065008 (2001); R. Lehnert, Phys. Rev. D 68, 085003 (2003). 
[10] H. Belich, M. A. De Andrade, M. A. Santos, Mod.Phys.Lett. A 20, 2305 (2005)H. Belich, T. Costa-Soares, M.M. Ferreira Jr., J. A. Helayël-Neto and M.T.D. Orlando, Int. J. Mod. Phys. A 21, 2415 (2006), A. P. Baeta Scarpelli and J. A. Helayel-Neto, Phys. Rev. D 73, 105020 (2006); N.M. Barraz, Jr., J.M. Fonseca, W.A. Moura-Melo, and J.A. Helayel-Neto, Phys. Rev. D76, 027701 (2007),H. Belich et al., Phys. Lett. A 370, 126 - 130 (2007), R. Casana, M. M. Ferreira,J. S. Rodrigues, Phys. Rev. D 78, 125013-1 (2008), R. Casana et al.,Phys. Rev. D 78, 105014 (2008), R. Casana et al.,Phys. Rev. D 78, 025030(2008), R. Casana et al.,Phys. Rev. D 80, 125040(2009), R. Casana et al., Phys. Rev. D 80, 085026(2009), R. Casana et al., Eur. Phys. J. C 62, 573-578, (2009), R. Casana et al., Phys. Rev. D 81, 105015, (2010).

[11] S.R. Coleman and S.L. Glashow, Phys. Rev. D 59, 116008 (1999); V. A. Kostelecky and M. Mewes, Phys. Rev. Lett. 87, 251304 (2001); V. A. Kostelecky and M. Mewes, Phys. Rev. D 66, 056005 (2002); J. Lipa et. al., Phys. Rev. Lett. 90, 060403 (2003).

[12] R. Bluhm, V.A. Kostelecky, and N. Russell, Phys. Rev. Lett. 79, 1432 (1997); R. Bluhm, V.A. Kostelecky, and N. Russell, Phys. Rev. D 57, 3932 (1998); R. Bluhm, V.A. Kostelecky, C. D. Lane, and N. Russell, Phys. Rev. Lett. 88, 090801 (2002); R. Bluhm and V.A. Kostelecky, Phys. Rev. Lett. 84, 1381 (2000); R. Bluhm, R. Bluhm, V.A. Kostelecky, and C. D. Lane, Phys. Rev. Lett. 84, 1098 (2000); V.A. Kostelecky, and N. Russell, Phys. Rev. Lett. 82, 2254 (1999); V.A. Kostelecky and C. Lane, Phys. Rev. D 60, 116010 (1999).

[13] V.A. Kostelecky and C. D. Lane, J. Math. Phys. 40, 6245 (1999); R. Lehnert, J. Math. Phys. 45, 3399 (2004).

[14] M.M. Ferreira Jr and F. M. O. Moucherek, Int. J. Mod. Phys. A 21, 6211 (2006); Nucl. Phys. A 790, 635 (2007); S. Chen, B. Wang, and R. Su, Class. Quant.Grav. 23, 7581,(2006);O. G. Kharlanov and V. Ch. Zhukovsky, J. Math. Phys. 48, 092302 (2007), M. Frank, I. Turan, I. Yurdusen, hep-th 07094276.

[15] H. Belich, T. Costa-Soares, M.M. Ferreira Jr., J. A. Helayël-Neto, and F. M. O. Moucherek, Phys. Rev. D 74, 065009 (2006).

[16] H. Muller, C. Braxmaier, S. Herrmann, and A. Peters, Phys. Rev. D 67, 056006 (2003); H. Müller, A. Saenz, A. Peters, and C. Lämmerzahl, Phys. Rev. D 68, 116006 (2003); H. Müller, Phys. Rev. D 71, 045004 (2005); N. Russell, Phys. Scripta 72, C38 (2005).

[17] D. F. Phillips, M. A. Humphrey, E. M. Mattison, R. E. Stoner, R. F C. Vessot, R. L. Walsworth, Phys.Rev. D63, 111101 (2001); D. Bear, R.E. Stoner, R.L. Walsworth, V. Alan Kostelecky, Charles D. Lane, Phys. Rev. Lett. 85, 5038 (2000); Erratum-ibid. 89 (2002) 209902; M.A. Humphrey, D.F. Phillips, R.L. Walsworth, Phys. Rev. A 68, 063807 (2003), J. Fonseca, A. Gomes, W.A. Moura-Melo, Phys Lett. B, 671, 280-283, (2009); A. H. Gomes, J. M. Fonseca, W. A. Moura-Melo, A. R. Pereira, JHEP 05, 104-1-104-11, (2010).

[18] V. A. Kostelecky and S. Samuel, Phys. Rev. Lett. 63, 224 (1989); Phys. Rev. Lett. 66, 1811 (1991); Phys. Rev. D 39, 683 (1989); Phys. Rev. D 40, 1886 (1989), V. A. Kostelecky and R. Potting, Nucl. Phys. B 359, 545 (1991); Phys. Lett. B 381, 89 (1996); V. A. Kostelecky and R. Potting, Phys. Rev. D 51, 3923 (1995).

[19] G. Barenboim, J.D. Lykken, arXiv:0908.2993.

[20] S. Esposito, G. Salesi, Mod. Phys. Lett. A 25, 597, (2010).

[21] D. Colladay and V. A. Kostelecký, Phys. Rev. D 55, 6760 (1997); D. Colladay and V. A. Kostelecký, Phys. Rev.D 58, 116002 (1998), V. A. Kostelecký and M. Mewes, Phys. Rev.D 69, 016005 (2004).

[22] M. S. Berger and V. A. Kostelecký, Phys.Rev. D65 , 091701 (2002); M. S. Berger, Phys. Rev. D 68, 115005 (2003) 
[23] P. A. Bolokhov, S. G. Nibbelink, M.Pospelov, Phys. Rev. D 72, 015013 (2005); S. G. Nibbelink and M. Pospelov, Phys. Rev. Lett. 94, 081601 (2005), S. G. Nibbelink, and T. S. Nyawelo, Phys. Rev. D 75, 045002 (2007).

[24] S.M. Carroll, G.B. Field and R. Jackiw, Phys. Rev. D 41, 1231 (1990).

[25] H. Belich et al., Phys. Rev. D 68, 065030 (2003); A. P. Baeta Scarpelli, H. Belich, J. L. Boldo, L. P. Colatto, J. A. Helayel-Neto and A. L. M. A. Nogueira, Nucl. Phys. Proc. Suppl. 127, 105 (2004).

[26] Q. G. Bailey, V.A. Kosteleck', Phys. Rev. D 70 (2004) 076006, G. Betschart, E. Kant, and F. R. Klinkhamer, Nucl. Phys. B, 815, 198-214 (2009).

[27] D. Mattingly, “Modern Test on Lorentz Invariance”, http://www.livingreviews.org/; LivingRev.Rel.8:5,(2005) .

[28] Gambini, R., and Pullin, J., "Nonstandard optics from quantum spacetime”, Phys. Rev. D, 59, 124021, (1999). Related online version (cited on 22 February 2005); http://arXiv.org/abs/gr-qc/9809038.

[29] H. Belich, L. G. Durand-Bernald, J. A Helayel-Neto, and F. J. L. Leal, work in progress.

[30] O.W. Greenberg, “CPT Violation Implies Violation of Lorentz Invariance”, Phys. Rev. Lett. 89, 231602 (2002).

[31] H. K. Dreiner, H. E. Haber, S. P. Martin, Two-component spinor techniques and Feynman rules for quantum field theory and supersymmetry, arXiv:0812.1594.

[32] A. Katz and Y. Shadmi, Phys. Rev. D 74, 115021 (2006). 